\title{
Flavobacterium johnsoniae Tyrosine Ammonia Lyase (FjTAL) in-silico Structure Prediction and Molecular Docking to L-Tyrosine, p-Coumaric Acid (pHCA) and Caffeic Acid
}

\author{
SeyyedAmirreza Mousavi Majd ${ }^{\mathrm{a}}$ \\ ${ }^{a}$ Department of Biotechnology, College of Science, University of Tehran, Tehran, Iran \\ Corresponding Email: $\underline{\text { sam00mb@gmail.com }}$
}

\begin{abstract}
Responsible for the conversion of L-tyrosine to p-coumaric acid in Flavobacterium johnsoniae, FjTAL has drawn the attention of many biochemical engineers who wish to carry out a sustainable biosynthetic scheme for the production of aromatic compounds. In this study, with the aid of various computational tools, the secondary and tertiary structures of FjTAL have been predicted. The results suggest that FjTAL forms a homo-tetramer when active as a cytosolic enzyme and it is mostly consisted of alpha helices. With the aid of molecular docking, one can hypothesize that FjTAL is likely to bind to L-tyrosine, p-coumaric acid, and caffeic acid with a similar molecular mechanism and thus, p-coumaric acid and caffeic acid may exhibit a negative feedback response toward the enzyme and inhibit its activity competitively. Two distinct binding pockets have been discovered, one of which contains highly conserved residues among several species. The residues which form the prosthetic group 3,5- dihydro-5methylidene-4H-imidazol-4-one (MIO) also emerge in the evolutionary conserved binding pocket. The other discovered cavity, could either be a second binding site for the ligands or simply an artifact of the molecular docking task.
\end{abstract}

Keywords: Protein Structure Prediction, Computational Biology, Tyrosine Ammonia Lyase (TAL), Flavobacterium johnsoniae Tyrosine Ammonia Lyase (FjTAL)

\section{Introduction}

\subsection{Background}

In the recent methods that have been developed for the sustainable production of phenylpropanoids in recombinant $E$. coli, FjTAL has been proposed as one of the potential enzymes to convert L-tyrosine to p-coumaric acid $^{12}$ (Fig.1). Although it has been demonstrated that Flavobacterium johnsoniae Tyrosine Ammonia Lyase (FjTAL), has satisfactory affinity for L-tyrosine $\left(\mathrm{K}_{\mathrm{m}} \sim 6.7\right.$ $\mu \mathrm{M})$, little has been reported on the binding affinity of other structurally-related metabolites to $\mathrm{FjTAL}^{3}{ }^{4}$. It has been hypothesized by Sariaslani ${ }^{5}$ (2007) and Haslinger and Prather (2020) that p-coumaric acid, the product of elimination reaction catalyzed by FjTAL, might inhibit the activity of the enzyme, exerting a kind of negative feedback response. The goal of this study, is to computationally predict the $3 \mathrm{D}$ structure of FjTAL, paving the way to help other researchers to reasonably engineer the protein for the future studies. It is worth mentioning that since not even a single experimental data on the structure of the enzyme in Protein Data Bank (PDB) was available, one had to only rely on computational methods in order to determine the structure of the enzyme.

\subsection{The in-silico Experiment Design}

The general strategy devised in this study is depicted in Fig.2. To predict the 3D structure of FjTAL, the sequence of the enzyme had to be retrieved. The codon-optimized DNA sequence of FjTAL was retrieved from the supplementary file of Haslinger and Prather $(2020)^{1}$, and translated it into amino acid alphabet. It was also observed that a record had already existed in NCBI with the exact sequence (SCV44818.1 unnamed protein product).

With the FjTAL protein sequence available, four webservers were employed in order to predict tertiary structure of FjTAL. Two of these 
webservers, SWISS-MODEL and Phyre2, implement homology modelling algorithms while the other two, AlphaFold2 and

RoseTTAFold, predict the structures without the aid of template (ab-initio prediction).

FjTAL protein sequence was fed into each of these four servers. Then, the quality of these structures was validated and the ones that did not meet the standards were eliminated. Eventually, the top five pdb files which were found to be superior among all, were stored as .pdb files for the next steps. After that, the secondary structures of the predicted models were extracted from the pdb files and secondary structure prediction methods that were only dependent on the input sequence rather than tertiary structure.

Fig.1 The artificial biosynthetic pathway developed by Rodrigues et al. $(2015)^{2}$ and Haslinger and Prather $(2020)^{1}$ and the structure of related metabolites at $\mathrm{pH}=7.4$

TAL: tyrosine ammonia lyase, e.g., FjTAL, RgTAL, SeSam8, RsTAL, HaTAL

*Many different enzymes are utilized for the next step of the artificial pathway including CYP199A2, 4-coumarate

3-hydroxylase, hydroxyphenylacetate 3-hydroxylase

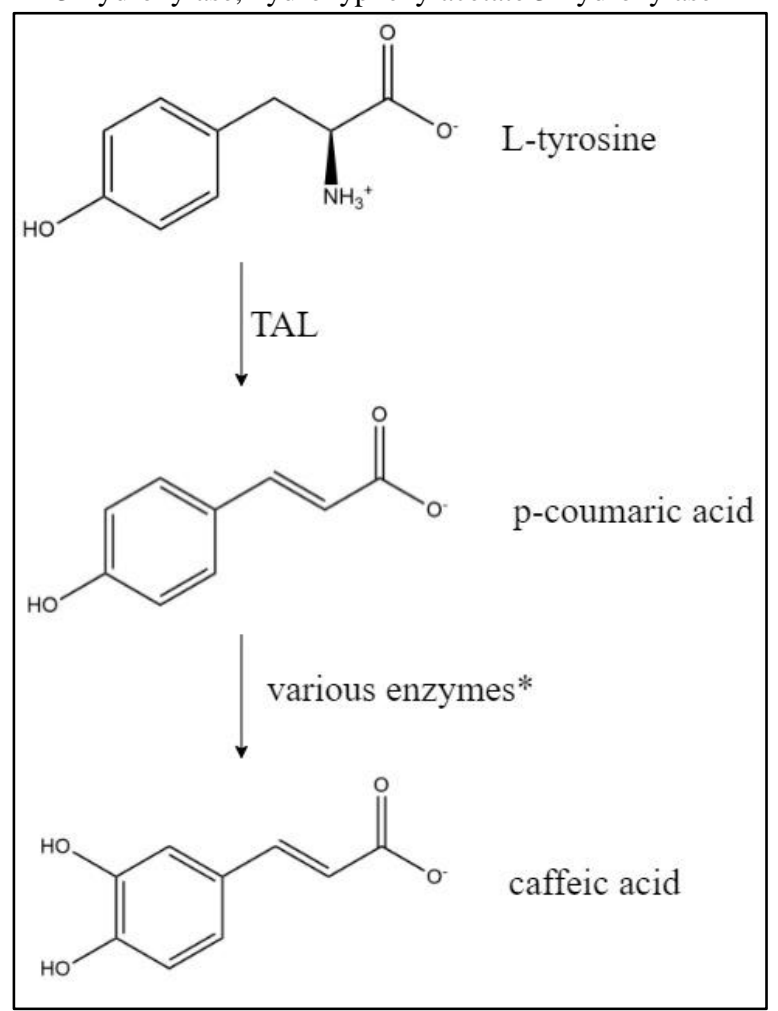

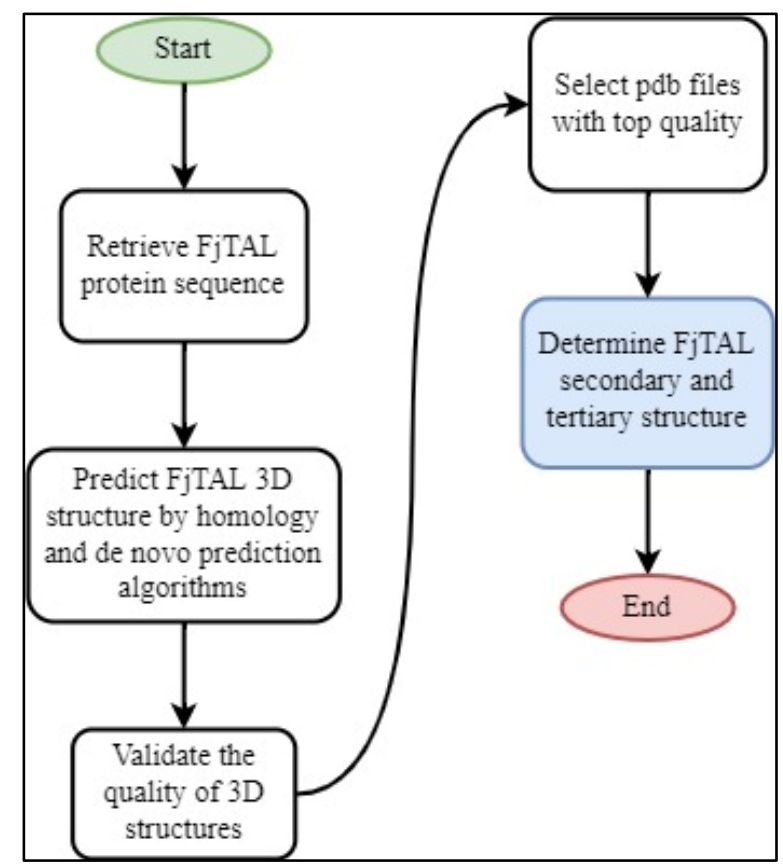

Fig.2 The workflow carried out in this study

\section{Materials and Methods}

In this study quite a few computational tools were employed to help us fulfil the computational experiment. The list of the software and webservers is given in Table1.

\begin{tabular}{|c|c|}
\hline Task & Database/Webserver/Software \\
\hline $\begin{array}{l}\text { DNA to AA } \\
\text { translation }\end{array}$ & Biostrings $(\mathrm{R})^{6}$ \\
\hline $\begin{array}{l}\text { Tertiary structure } \\
\text { prediction }\end{array}$ & 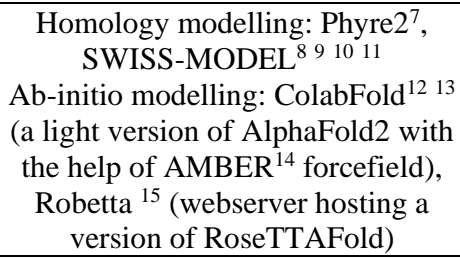 \\
\hline $\begin{array}{c}\text {.pdb file quality } \\
\text { assessment }\end{array}$ & SAVES of UCLA ${ }^{161718}$ \\
\hline $\begin{array}{c}\text { Secondary } \\
\text { structure retrieval } \\
\text { from .pdb files }\end{array}$ & Stride $^{19}$ \\
\hline $\begin{array}{l}\text { Secondary } \\
\text { structure } \\
\text { prediction } \\
\text { (sequence input } \\
\text { only) }\end{array}$ & JPred $4^{20}$, PROTEUS2 $2^{21}$ \\
\hline $\begin{array}{c}\text { Superimpose } \\
\text { structures and } \\
\text { calculate RMSD }\end{array}$ & BIOVIA Discovery Studio \\
\hline .pdb file viewer & Chimera $\mathrm{X}^{22}{ }^{23}, \mathrm{Mol}^{* 24}$ \\
\hline $\begin{array}{l}\text { Ligands .mol } \\
\text { files retrieval }\end{array}$ & ChemSpider \\
\hline
\end{tabular}




\begin{tabular}{|c|c|}
\hline $\begin{array}{c}\text { Add/Remove } \\
\text { proton to ligands } \\
. \text { mol files } \\
(\mathrm{pH}=7.4)\end{array}$ & $\begin{array}{c}\text { Galaxy servers }^{25}{ }^{26},{\text { Open } \text { Babel }^{27}} \\
\text { Pybel }^{28}\end{array}$ \\
\hline $\begin{array}{l}\text { Ligands MM2 } \\
\text { minimization }\end{array}$ & Chem3D \\
\hline $\begin{array}{c}\text { Blind molecular } \\
\text { docking }\end{array}$ & $\begin{array}{l}\text { CB-Dock }{ }^{29}{ }^{30} \text {, a blind docking tool } \\
\text { based on AutoDock Vina }{ }^{31}\end{array}$ \\
\hline Miscellaneous & $\begin{array}{c}\text { ChemDraw }^{32} \text {, draw.io, GraphPad } \\
\text { Prism }\end{array}$ \\
\hline
\end{tabular}

Table1 the list of computational tools used throughout this survey.

\section{Results}

\subsection{FjTAL Structure Prediction and Quality Assesment}

As mentioned before, four webservers were employed to predict the structure of FjTAL. Phyre2, SWISS-MODEL, ColabFold and Robetta generate one, two, five and five structures respectively. It is worth noting that ColabFold initially generates five unrelaxed structures and five AMBER-relaxed structures which we used only the latter for the purposes of this study. SWISS-MODEL also predicted that FjTAL is likely to be either a homotetrameric or a homodimeric protein. However, most of the closely-related homologs of FjTAL were proven to be homotetrameric and thus, we have concluded this may also be a valid assumption for FjTAL as well.

All thirteen pdb files, were passed to SAVES webserver to assess the quality of the predicted structures. The ideal structures had to pass Verify3D test and achieve a score $>95$ in ERRAT program (top five structures were selected). As shown in Table2, ab-initio algorithms significantly outperformed Phyre2 and SWISS-MODEL. The superiority of AlphaFold2 and RoseTTAFold over classical methods had also been proposed in other studies such as $\mathrm{CAMEO}^{33}{ }^{34}$ as well. The top five structures were selected and stored.

\footnotetext{
Table 2 The results of quality assessment using SAVES.

FjTAL_Final_Phyre2.pdb is the file generated on Phyre2 server. FjTAL_X_SWISS.pdb files are the output of SWISS-MODEL. FjTAL_X_Rob.pdb files are the tertiary structures predicted by Robetta/RoseTTAFold.
}

FjTAL_X_AF2 files are the output of ColabFold/AlphaFold2.

\begin{tabular}{lll} 
& ERRAT & VERIFY \\
\hline FjTAL_Final_Phyre2 & 79.5181 & PASS \\
\hline FjTAL_2_SWISS & 89.7918 & PASS \\
\hline FjTAL_1_SWISS & 89.9897 & FAIL \\
\hline FjTAL_5_Rob & 93.7374 & PASS \\
FjTAL_1_Rob & 94.5122 & PASS \\
\hline FjTAL_2_Rob & 95.3157 & PASS \\
FjTAL_2_AF2 & 95.6701 & PASS \\
FjTAL_5_AF2 & 96.0986 & PASS \\
\hline FjTAL_3_Rob & 96.5726 & PASS \\
FjTAL_4_AF2 & 97.9381 & PASS \\
FjTAL_1_AF2 & 97.9508 & PASS \\
FjTAL_4_Rob & 97.9633 & PASS \\
FjTAL_3_AF2 & 98.5714 & PASS
\end{tabular}

\subsection{FjTAL Secondary and Tertiary Structure}

The top models from previous sections (Table2 yellow highlights) were passed to Stride to extract the secondary structures from pdb files.

We also tried to predict the secondary structures using JPred4 and PROTEUS2 webservers which require the protein sequence as their only input.

In Table 3 the prediction results by JPred4, PROTEUS2 and the extraction results by Stride are tabulated.

\begin{tabular}{|c|c|c|c|c|c|c|c|}
\hline & FịAL_1_AF2 (Stride) & FiTAL_3_AF2 (Stride) & FijAL_3_Rob (Stride) & FiTAL_4_AF2 (Stride) & FiTAL_4_Rob (Stride) & Sequence (JPred4) & Sequence (PROTEUS2) \\
\hline \# of alpha helices & 19 & 19 & 18 & 19 & 18 & 17 & 18 \\
\hline \# of 310 helices & 4 & 5 & 5 & 5 & 5 & $\mathrm{NA}$ & $\mathrm{NA}$ \\
\hline \# of beta strands & 9 & 9 & 7 & 9 & 5 & 10 & 16 \\
\hline
\end{tabular}


bioRxiv preprint doi: https://doi.org/10.1101/2022.02.09.479702; this version posted February 14, 2022. The copyright holder for this preprint (which was not certified by peer review) is the author/funder, who has granted bioRxiv a license to display the preprint in perpetuity. It is made available under aCC-BY-ND 4.0 International license.

As shown in Table3, while, to some extents, the results differ from one model to another, one can state that FjTAL is mostly consisted of alpha helices with very few, short length, beta strands.

Note that JPred4 and PROTEUS2 do not generate any data on the number of 310 helices that may exist in the structures and also notice that the number of beta strands differ significantly in each record, mainly because FjTAL seems to have very short (length $=<4$ amino acid) beta strands. This has led to emergence of presumably false positive results in the case of beta strands especially in the prediction-based results (See Fig.3 and Fig.4).

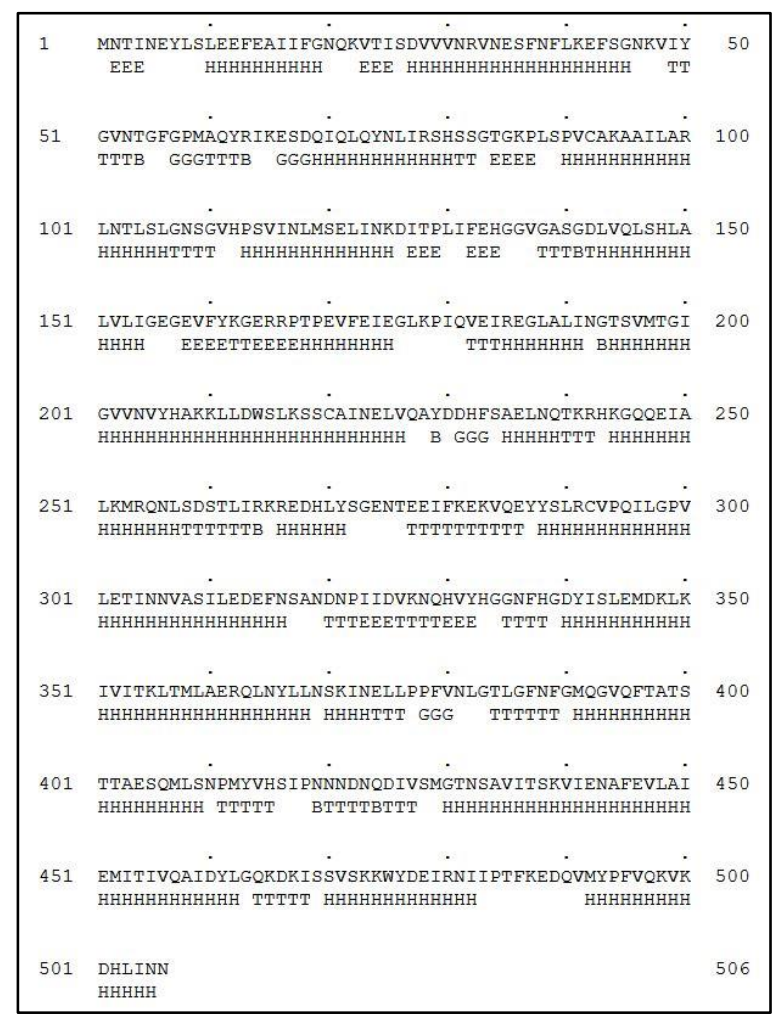

Fig.3 The secondary structures retrieved from FjTAL_1_AF2 as one of the top candidate structures.

H: Alpha helix, G: 3-10 helix, I: PI-helix, E: Extended conformation (beta strand), B: Isolated bridge, T: Turn, C: Coil (none of the above)

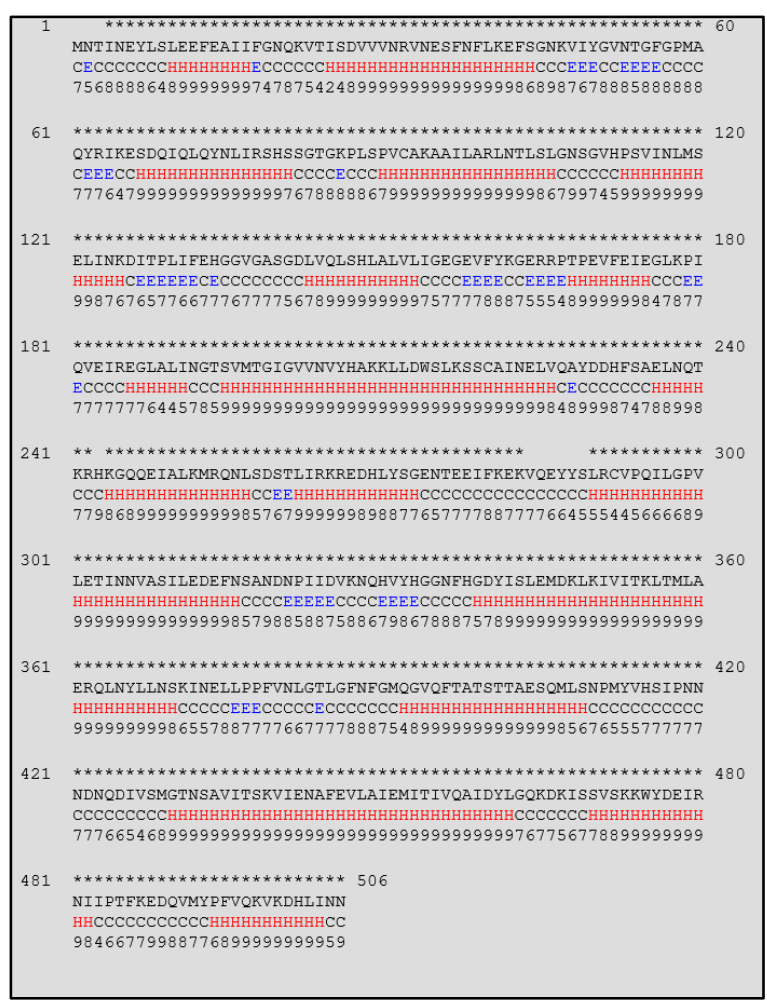

Fig.4 Secondary structures predicted by PROTEUS2. (C: coil, E: beta strand, H: alpha helix)

The structure of FjTAL_1_AF2 is shown in Fig.5 as an example.

We also passed the pdb files to BIOVIA Discovery Studio in order to calculate RMSD, as a measure of similarity among the structures (see Table4 and Fig.6).

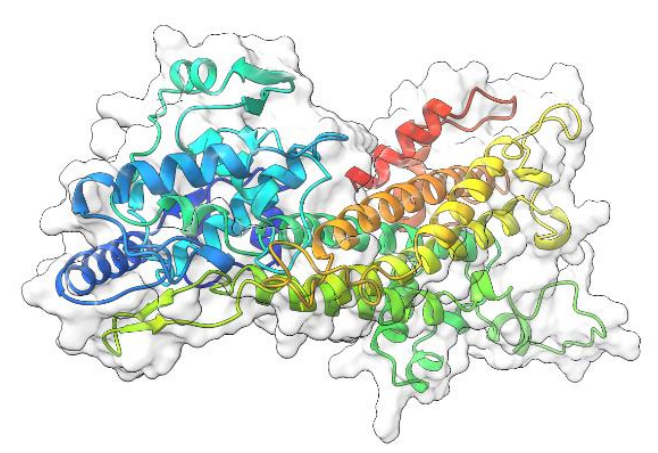

Fig.5 Ribbon visualization of FjTAL_1_AF2.pdb as one of the candidate structures for FjTAL. 


\begin{tabular}{|c|c|c|c|c|c|}
\hline Molecule & Reference & C-Alpha & Main-chain & Side-chain & $\begin{array}{c}\text { All } \\
\text { Protein }\end{array}$ \\
\hline $\begin{array}{l}\text { FJTAL_3 } \\
\text { AF2 }\end{array}$ & FjTAL_1_AF2 & 0.237 & 0.285 & 0.67 & 0.512 \\
\hline $\begin{array}{l}\text { FjTAL_3 } \\
\text { Rob }\end{array}$ & FjTAL_1_AF2 & 1.456 & 1.48 & 2.608 & 2.112 \\
\hline $\begin{array}{l}\text { FjTAL_4_ } \\
\text { AF2 }\end{array}$ & FjTAL_1_AF2 & 0.276 & 0.313 & 0.736 & 0.563 \\
\hline $\begin{array}{l}\text { FJTAL_4_ } \\
\text { Rob }\end{array}$ & FjTAL_1_AF2 & 1.423 & 1.447 & 2.608 & 2.1 \\
\hline
\end{tabular}

Table4 RMSD values for each pair of models. We have arbitrarily taken FjTAL_1_AF2 as the reference model.

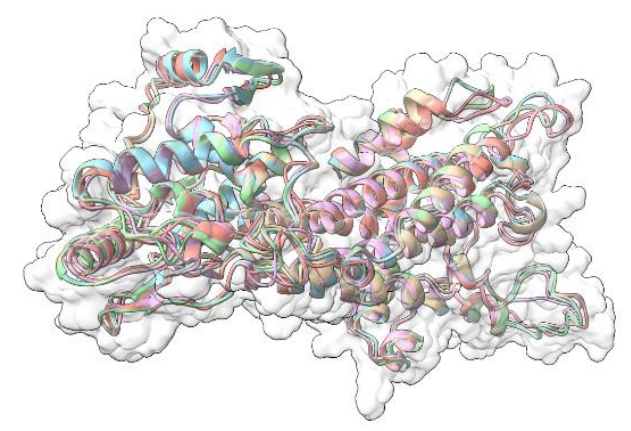

Fig.6 The superimposed structure of FjTAL

Note that there exists a significant difference $(\triangle \mathrm{RMSD}>2$ Angstrom) in the structures predicted by Robetta/RoseTTAFold and ColabFold/AlphaFold2. Their difference mainly stems from the coils and turns rather than the alpha helices. Without the availability of an experimentally-verified 3D structure for the enzyme, such workflow was the only logical way one could turn into to find out more about the structure of FjTAL and its relevance to its function.

\subsection{FjTAL Molecular Docking to L-Tyrosine, p- Coumaric Acid and Caffeic Acid}

With the availability of the predicted structure of FjTAL, a molecular docking task was executed. The structures of ligands i.e., L-tyrosine, $p$ coumaric acid and caffeic acid were retrieved from ChemSpider and their structures were subsequently adjusted to $\mathrm{pH}=7.4$. Then, an MM2 minimization task was performed to retrieve the best energetically-favored representation of the ligands. The five receptor pdb files were docked with three potential ligands, L-tyrosine, p-coumaric acid and caffeic acid via the vina-based docking server, CBDock.

CB-Dock reports five cavities and their corresponding vina score for each pair of ligand and receptor. The top results, all of which possessing a vina score $=<-5.5$, were selected for further investigations.

We used mol* to determine the residues that constituted the binding pocket of FjTAL in each of these complexes. Interestingly two distinct binding sites, named after their first residues as VQAY and VIY cavities, consistently appeared in most of the structures (see Table 5, Fig.7 and Fig.8).

Some of the residues that constitute the VIY cavity, are known to form the prosthetic group, 3,5- dihydro-5-methylidene-4H-imidazol-4-one (MIO), which has been proved to act as an electrophilic species in the deamination reaction catalyzed by Phenylalanine Ammonia Lyase (PAL). ${ }^{35}$ VIY cavity homologs also appeared in the experimentally-verified structure of Rhodobacter sphaeroides Tyrosine Ammonia Lyase, RsTAL in complex with caffeate and coumarate $^{36}$. The VQAY cavity exhibits very few evolutionary-conserved residues, suggesting that it is either completely an artifact or it could be another binding site to the ligands as well as the VIY cavity. Site directed mutagenesis studies can help us further illustrate the importance of VQAY cavity.

The molecular docking results demonstrate that pHCA and caffeic acid bind to FjTAL in the same pocket as L-tyrosine, supporting the idea that these two may competitively inhibit the activity of FjTAL, and further result in a negative feedback response. ${ }^{5}$ 
bioRxiv preprint doi: https://doi.org/10.1101/2022.02.09.479702; this version posted February 14,2022 . The copyright holder for this preprint (which was not certified by peer review) is the author/funder, who has granted bioRxiv a license to display the preprint in perpetuity. It is made available under aCC-BY-ND 4.0 International license.

\begin{tabular}{|c|c|c|c|c|c|c|c|}
\hline \multirow{2}{*}{\multicolumn{2}{|c|}{$\begin{array}{l}\text { Table format: } \\
\text { Grouped }\end{array}$}} & \multirow{2}{*}{\multicolumn{2}{|c|}{$\begin{array}{l}\text { Group A } \\
\text { L-tyrosine }\end{array}$}} & \multirow{2}{*}{\multicolumn{2}{|c|}{$\begin{array}{c}\text { Group B } \\
\text { p-coumaric acid }\end{array}$}} & \multirow{2}{*}{\multicolumn{2}{|c|}{$\begin{array}{l}\text { Group C } \\
\text { caffeic acid }\end{array}$}} \\
\hline & & & & & & & \\
\hline 4 & 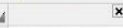 & A:1 & A:2 & B:1 & B:2 & $c: 1$ & $C: 2$ \\
\hline 1 & FJTAL_1_AF2 & -6.2 & -5.7 & -5.7 & -5.6 & -5.9 & -5.8 \\
\hline 2 & FJTAL___AF2 & -6.0 & -5.7 & -5.6 & -5.6 & -5.8 & -5.8 \\
\hline 3 & FJTAL_3_Rob & -5.7 & & -6.0 & & -6.1 & -5.6 \\
\hline 4 & FJTAL_4_AF2 & -6.1 & -5.7 & -5.7 & -5.6 & -5.9 & -5.6 \\
\hline 5 & FjTAL_4_Rob & -5.5 & & -5.5 & & -5.7 & \\
\hline
\end{tabular}

Table 5 The molecular docking top results and their corresponding binding cavities. VQAY cavity is in blue and VIY cavity is in red. Other colors represent rare and presumably false binding sites.

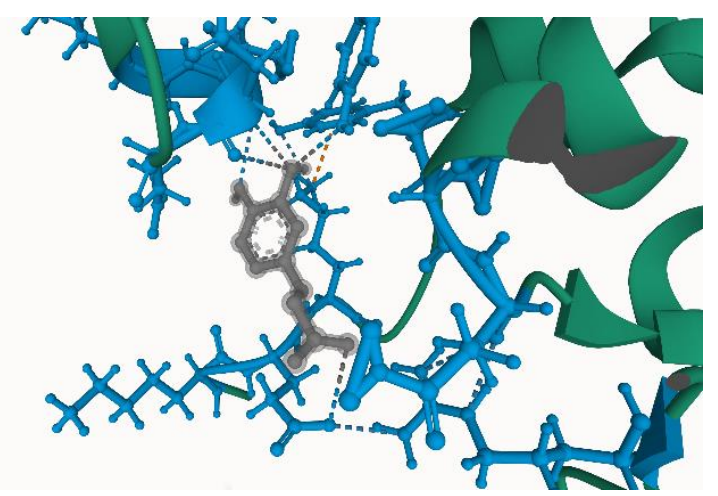

Fig.7 The predicted binding site of $\mathrm{pH}$-adjusted, MM2 minimized, caffeic acid and FJTAL_1_AF2.pdb with vina score $=-5.8$

The blue residues depict the VQAY pocket, which is comprised of less evolutionary conserved residues.

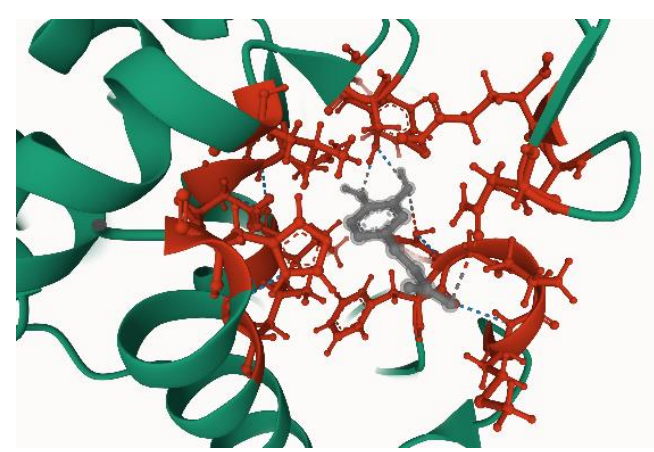

Fig. 8 The predicted binding site of $\mathrm{pH}$-adjusted, MM2 minimized, caffeic acid and FjTAL_1_AF2.pdb with vina score $=-5.9$

The red residues depict the VIY pocket, consisted of many evolutionary conserved residues.

\section{Supplementary material}

The PDB files of the predicted structures, ligands and docking results are uploaded.

\section{References}

1. Haslinger, K. \& Prather, K. L. J. Heterologous caffeic acid biosynthesis in Escherichia coli is affected by choice of tyrosine ammonia lyase and redox partners for bacterial Cytochrome P450. Microb. Cell Fact. 19, 26 (2020).

2. Rodrigues, J. L., Araújo, R. G., Prather, K. L. J., Kluskens, L. D. \& Rodrigues, L. R. Heterologous production of caffeic acid from tyrosine in Escherichia coli. Enzyme Microb. Technol. 71, 3644 (2015).

3. Jendresen, C. B. et al. Highly Active and Specific Tyrosine Ammonia-Lyases from Diverse Origins Enable Enhanced Production of Aromatic Compounds in Bacteria and Saccharomyces cerevisiae. Appl. Environ. Microbiol. 81, 44584476 (2015).

4. Xue, Z. et al. Improved production of phydroxycinnamic acid from tyrosine using a novel thermostable phenylalanine/tyrosine ammonia lyase enzyme. Enzyme Microb. Technol. 42, 58-64 (2007).

5. Sariaslani, F. S. Development of a Combined Biological and Chemical Process for Production of Industrial Aromatics from Renewable Resources. Annu. Rev. Microbiol. 61, 51-69 (2007).

6. Pagès, H., Aboyoun, P., Gentleman, R. \& DebRoy, S. Biostrings: Efficient manipulation of biological strings. (2020).

7. Kelley, L. A., Mezulis, S., Yates, C. M., Wass, M. N. \& Sternberg, M. J. E. The Phyre2 web portal for protein modeling, prediction and analysis. Nat. Protoc. 10, 845-858 (2015).

8. Bienert, S. et al. The SWISS-MODEL Repository - new features and functionality. Nucleic Acids Res. 45, D313-D319 (2017).

9. Waterhouse, A. et al. SWISS-MODEL: homology modelling of protein structures and complexes. Nucleic Acids Res. 46, W296-W303 (2018).

10. Benkert, P., Biasini, M. \& Schwede, T. Toward the estimation of the absolute quality of individual protein structure models. Bioinformatics 27, 343350 (2011).

11. Mariani, V., Biasini, M., Barbato, A. \& Schwede, T. IDDT: a local superposition-free score for comparing protein structures and models using distance difference tests. Bioinformatics 29, 27222728 (2013).

12. Mirdita, M. et al. ColabFold - Making protein folding accessible to all. bioRxiv 
2021.08.15.456425 (2021)

doi:10.1101/2021.08.15.456425.

13. Jumper, J. et al. Highly accurate protein structure prediction with AlphaFold. Nature 596, 583-589 (2021).

14. Case, D. A. et al. Amber 2021. (University of California, San Francisco, 2021).

15. Minkyung, B. et al. Accurate prediction of protein structures and interactions using a three-track neural network. Science (80-. ). 373, 871-876 (2021).

16. Lüthy, R., Bowie, J. U. \& Eisenberg, D. Assessment of protein models with threedimensional profiles. Nature 356, 83-85 (1992).

17. Bowie, J. U., Lüthy, R. \& Eisenberg, D. A method to identify protein sequences that fold into a known three-dimensional structure. Science $\mathbf{2 5 3}$, 164-170 (1991).

18. Colovos, C. \& Yeates, T. O. Verification of protein structures: patterns of nonbonded atomic interactions. Protein Sci. 2, 1511-1519 (1993).

19. Frishman, D. \& Argos, P. Knowledge-based protein secondary structure assignment. Proteins Struct. Funct. Bioinforma. 23, 566-579 (1995).

20. Drozdetskiy, A., Cole, C., Procter, J. \& Barton, G. J. JPred4: a protein secondary structure prediction server. Nucleic Acids Res. 43, W389-W394 (2015).

21. Montgomerie, S. et al. PROTEUS2: a web server for comprehensive protein structure prediction and structure-based annotation. Nucleic Acids Res. 36, W202-W209 (2008).

22. Goddard, T. D. et al. UCSF ChimeraX: Meeting modern challenges in visualization and analysis. Protein Sci. 27, 14-25 (2018).

23. Pettersen, E. F. et al. UCSF ChimeraX: Structure visualization for researchers, educators, and developers. Protein Sci. 30, 70-82 (2021).

24. Sehnal, D. et al. Mol* Viewer: modern web app for 3D visualization and analysis of large biomolecular structures. Nucleic Acids Res. 49, W431-W437 (2021).

25. Afgan, E. et al. The Galaxy platform for accessible, reproducible and collaborative biomedical analyses: 2018 update. Nucleic Acids Res. 46, W537-W544 (2018).

26. Bray, S. A., Lucas, X., Kumar, A. \& Grüning, B. A. The ChemicalToolbox: reproducible, userfriendly cheminformatics analysis on the Galaxy platform. J. Cheminform. 12, 40 (2020).

27. chemical toolbox. J. Cheminform. 3, 33 (2011).

28. O’Boyle, N. M., Morley, C. \& Hutchison, G. R. Pybel: a Python wrapper for the OpenBabel cheminformatics toolkit. Chem. Cent. J. 2, 5 (2008).

29. Liu, Y. et al. CB-Dock: a web server for cavity detection-guided protein-ligand blind docking. Acta Pharmacol. Sin. 41, 138-144 (2020).

30. Cao, Y. \& Li, L. Improved protein-ligand binding affinity prediction by using a curvature-dependent surface-area model. Bioinformatics 30, 1674-1680 (2014).

31. Trott, O. \& Olson, A. J. AutoDock Vina: Improving the speed and accuracy of docking with a new scoring function, efficient optimization, and multithreading. J. Comput. Chem. 31, 455-461 (2010).

32. Cousins, K. R. ChemDraw Ultra 9.0. CambridgeSoft, 100 CambridgePark Drive, Cambridge, MA 02140. www. cambridgesoft.com. See Web site for pricing options. J. Am. Chem. Soc. 127, 4115-4116 (2005).

33. Haas, J. et al. Introducing "best single template" models as reference baseline for the Continuous Automated Model Evaluation (CAMEO). Proteins Struct. Funct. Bioinforma. 87, 1378-1387 (2019).

34. Robin, X. et al. Continuous Automated Model EvaluatiOn (CAMEO)-Perspectives on the future of fully automated evaluation of structure prediction methods. Proteins Struct. Funct. Bioinforma. 89, 1977-1986 (2021).

35. Calabrese, J. C., Jordan, D. B., Boodhoo, A., Sariaslani, S. \& Vannelli, T. Crystal Structure of Phenylalanine Ammonia Lyase: Multiple Helix Dipoles Implicated in Catalysis,. Biochemistry 43, 11403-11416 (2004).

36. Louie, G. V et al. Structural Determinants and Modulation of Substrate Specificity in Phenylalanine-Tyrosine Ammonia-Lyases. Chem. Biol. 13, 1327-1338 (2006). 